\title{
Diagram technique for the Hubbard model. Ladder diagram summation
}

\author{
V.A.Moskalenko ${ }^{1,2}$, L.Z.Kon ${ }^{2}$ \\ 1 Joint Institute for Nuclear Research, 141980 Dubna, Russia \\ 2 Institute of Applied Physics, 5 Grosul St., 277028 Kishinev, Moldova
}

Received March 20, 1998

\begin{abstract}
A new diagram technique based on the generalized Wick theorem has been elaborated for the systems with strong electronic correlations. Coulomb repulsion of the electrons of the Hubbard model is considered as the main part of Hamiltonian and is taken into account in a zero order approximation. The hopping matrix elements are considered as a perturbation. One-particle Matsubara-Green function of the model has been investigated and Dyson equation has been obtained. New elements of the theory which are characteristic of this new approach are the local many-particle irreducible Green functions, or Kubo cumulants. They become zero when Coulomb interaction is zero. The main task of this paper is the summing of ladder diagrams which take into account the most essential charge and spin fluctuations of the system. The integral equations, which sum such diagrams, have been established for two different channels. The coherent potential approximation has been used to simplify and solve these equations. On this basis a metal-dielectric phase transition has been investigated.
\end{abstract}

Key words: generalized Wick theorem, ladder diagrams, Hubbard model

PACS: $71.28 .+d, 71.27 .+a$

\section{Introduction}

In the previous papers [1-5] a new diagram technique for the systems with strong electron correlations was elaborated. Such systems contain as the main part of their Hamiltonian on-site Coulomb repulsion of electrons. This interaction has to be taken into account in the zero order Hamiltonian of such systems. The other terms of the Hamiltonian, such as the hopping matrix elements of the Hubbard model [6] or hybridization of electron states of the periodic Anderson model [7] are treated as perturbations.

Because the zero order Hamiltonian which contains the above mentioned Coulomb interaction can be diagonalized by using the Hubbard and not a free electron operators, the ordinary Wick theorem proposed in the weak coupling field theory 
for disentangling chronological averages of an electron operator is not valid. Instead of a weak coupling, the Generalized Wick Theorem (GWT) has been proposed for the first time for a one-band Hubbard model with the Hamiltonian

$$
\begin{gathered}
H=H^{0}+H_{i n t}, H^{0}=\sum_{i} H_{i}^{0}, H_{i}^{0}=-\mu \sum_{\sigma} n_{i \sigma}+U n_{i_{\uparrow}} n_{i_{\downarrow}}, \\
H_{i n t}=\sum_{i j^{\sigma}} t(j-i) c_{j \sigma}^{+} c_{i \sigma}, \quad n_{i \sigma}=c_{i \sigma}^{+} c_{i \sigma},
\end{gathered}
$$

where $\mathrm{c}_{j \sigma}^{+}\left(c_{j \sigma)}\right.$ are an electron creation (annihilation) operator with spin $\sigma$ and site index $j$. Here $U$ is Coulomb repulsion of an electron, $\mu$ - a chemical potential of the system and $t(j-i)$ - a matrix element of hopping.

As the zero order density matrix is factorized on the site indices, all the calculations of the thermodynamic perturbation theory contributions are made in the local presentation. The sum of such contributions gives us the effect of delocalization and renormalization of the dynamical functions.

The new elements of the GWT are one-site many-particle irreducible Green functions $G_{n}^{(0) i r}$ or Kubo cumulants. These quantities contain all spin and charge fluctuations of a strong correlated system. They are identically equal to zero when Coulomb interaction is zero.

The next simple example can be useful to elucidate this question. We apply the GWT to the following chronological average:

$$
\begin{gathered}
\left\langle T c\left(x_{1}\right) c\left(x_{2}\right) \bar{c}\left(x_{3}\right) \bar{c}\left(x_{4}\right)\right\rangle_{0}=\left\langle T c\left(x_{1}\right) \bar{c}\left(x_{4}\right)\right\rangle_{0}\left\langle T c\left(x_{2}\right) \bar{c}\left(x_{3}\right)\right\rangle_{0}- \\
-\left\langle T c\left(x_{1}\right) \bar{c}\left(x_{3}\right)\right\rangle_{0}\left\langle T c\left(x_{2}\right) \bar{c}\left(x_{4}\right)\right\rangle_{0}+G_{2}^{(0) i r}\left[x_{1} x_{2} \mid x_{3} x_{4}\right] .
\end{gathered}
$$

Here $x$ stands for $\boldsymbol{x}, \sigma, \tau$.

All the quantities of the right-hand side of this equation are local. The zero order or the local approximation of the Matsubara one-particle Green function is equal to

$$
\begin{gathered}
G_{1}^{(0)}\left(x_{1} x_{2}\right)=-\left\langle T c\left(x_{1}\right) \bar{c}\left(x_{2}\right)\right\rangle_{0}=\delta_{\boldsymbol{x}_{1}, \boldsymbol{x}_{2}} G_{\sigma_{1} \sigma_{2}}^{(0)}\left(\tau_{1}-\tau_{2}\right) \\
G_{\sigma_{1} \sigma_{2}}^{(0)}\left(\tau_{1}-\tau_{2}\right)=-\left\langle T c_{\sigma_{1}}\left(\tau_{1}\right) \bar{c}_{\sigma_{2}}\left(\tau_{2}\right)\right\rangle_{0}
\end{gathered}
$$

where $\mathrm{c}(\tau)$ and $\bar{c}(\tau)$ are interaction presentations of electron operators and $\tau$ imaginary time of thermodynamic Green functions. Symbol $\langle\ldots\rangle_{0}$ stands for the statistical average with a zero order density matrix. A two-particle irreducible Green function $G_{2}^{(0) i r}$ is a local quantity:

$$
G_{2}^{(0) i r}\left[x_{1}, x_{2} \mid x_{3} x_{4}\right]=\delta_{\boldsymbol{x}_{1} \boldsymbol{x}_{2}} \delta_{\boldsymbol{x}_{1} \boldsymbol{x}_{3}} \delta_{\boldsymbol{x}_{1} x_{4}} G_{2}^{(0) i r}\left[\sigma_{1}, \tau_{1} ; \sigma_{2} \tau_{2} \mid \sigma_{3}, \tau_{3} ; \sigma_{4}, \tau_{4}\right]
$$

with the structure of Kubo cumulant:

$$
\begin{gathered}
G_{2}^{(0) i r}\left[\sigma_{1}, \tau_{1} ; \sigma_{2}, \tau_{2} \mid \sigma_{3}, \tau_{3} ; \sigma_{4}, \tau_{4}\right]=\left\langle T c_{\sigma_{1}}\left(\tau_{1}\right) c_{\sigma_{2}}\left(\tau_{2}\right) \bar{c}_{\sigma_{3}}\left(\tau_{3}\right) \bar{c}_{\sigma_{4}}\left(\tau_{4}\right)\right\rangle_{0}- \\
-\left\langle T c_{\sigma_{1}}\left(\tau_{1}\right) \bar{c}_{\sigma_{4}}\left(\tau_{4}\right)\right\rangle_{0}\left\langle T c_{\sigma_{2}}\left(\tau_{2}\right) \bar{c}_{\sigma_{3}}\left(\tau_{3}\right)\right\rangle_{0}+\left\langle T c_{\sigma_{1}}\left(\tau_{1}\right) \bar{c}_{\sigma_{3}}\left(\tau_{3}\right)\right\rangle_{0}\left\langle T c_{\sigma_{2}}\left(\tau_{2}\right) \bar{c}_{\sigma_{4}}\left(\tau_{4}\right)\right\rangle_{0}
\end{gathered}
$$


This quantity is just a new element of the GWT because the first two terms of the right-hand side of equation (2) are the ordinary Wick theorem contributions. The signs of these terms, and in general of all of them, are determined by the number of permutation $P$ which is necessary to obtain the final from the initial order of electron operators. The Hubbard operators appear only at the last stage of such an investigation - when the local quantities are calculated.

In the Generalized Wick theorem for Hubbard operators $X_{i}^{n m}$, used in papers [8-10], the electrons operators are expressed with their aid at the initial stage of the investigation. Because the Hubbard operators have a more complicated algebraical structure, the diagram technique for them is more complicated.

Now we shall consider our approach. In the higher orders of the perturbation theory there appear more complicated irreducible structures. For example, irreducible functions $G_{n}^{(0) i r}$ with the number of particles $n=3,4 \ldots$ will appear. Then, different products of irreducible functions $G_{m_{1}}^{(0) i r} \mathrm{G}_{m_{2}}^{(0) i r} G_{m_{3}}^{(0) i r}$ will be obtained, where the sum $m_{1}+m_{2}+m_{3}=n$ is equal to the number of particles that participate in the process of delocalization.

We can formulate the GWT in the following form:

$$
\begin{gathered}
G_{n}^{(0)}\left(x_{1} \ldots x_{n} \mid x_{1}^{\prime} \ldots x_{n}^{\prime}\right)=(-1)^{n}\left\langle T c\left(x_{1}\right) \ldots c\left(x_{n}\right) \bar{c}\left(x_{1}^{\prime}\right) \ldots \bar{c}\left(x_{n}^{\prime}\right)\right\rangle_{0}= \\
\sum_{\{P\}}(-1)^{P} G^{(0)}\left(x_{1} \mid x_{1}^{\prime}\right) \ldots G^{(0)}\left(x_{n} \mid x_{n}^{\prime}\right)+\sum_{\{P\}}(-1)^{P} \sum_{\substack{m_{1} \geqslant 1, m_{2} \geqslant 1, m_{1}+m_{2}+\ldots=n}} G_{m_{1}}^{(0) i r}\left[x_{1} \ldots x_{m_{1}} \mid x_{1}^{\prime} \ldots x_{m_{1}}^{\prime}\right] \times \\
\times G_{m_{2}}^{(0) i r}\left[x_{m_{1}+1 \ldots} x_{m_{1}+m_{2}} \mid x_{m_{1}+1 \ldots}^{\prime} x_{m_{1}+m_{2}}^{\prime}\right]+G_{n}^{(0) i r}\left[x_{1} \ldots x_{n} \mid x_{1}^{\prime} \ldots x_{n}^{\prime}\right] .
\end{gathered}
$$

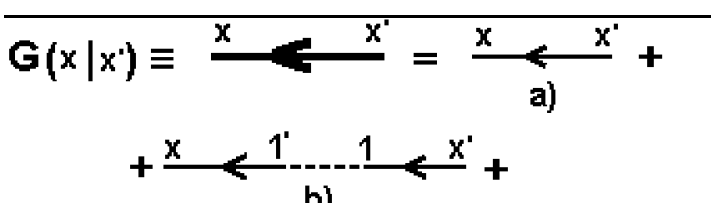

b)
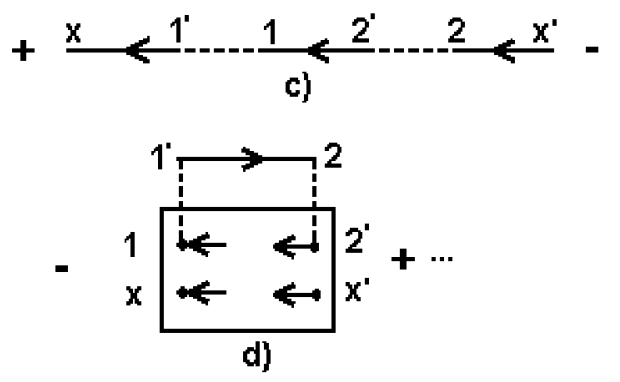

Figure 1. Diagrams of the first two orders of the perturbation theory for oneparticle Green-function. Diagram a) is of the zero order, b) of the first and c) and d) of the second order contributions. a), b) and c) diagrams are of a chain type and d) of a new kind, containing the first Kubo cumulant $G_{2}^{(0) i r}$.
In (5) we mean that $G_{1}^{(0) i r}\left(x_{i} \mid x_{i}^{\prime}\right)=$ $G^{(0)}\left(x_{i} \mid x_{i}^{\prime}\right)$.

The first term of the right-hand side of (5) is of a usual Wick kind, but all the next ones are characteristic of strongly correlated systems.

The diagrammatic rules for writing down the contributions of the perturbation theory series have been formulated in [1-2]. One-particle Green function $G^{(0)}\left(x_{1} \mid x_{1}^{\prime}\right)$ is represented by a thin solid line directed from $x_{1}^{\prime}$ to $x_{1}$ and the local quantity

$G_{n}^{(0) i r}\left[x_{1} \ldots x_{n} \mid x_{1}^{\prime} \ldots x_{n}^{\prime}\right]$ - by a rectangle which surrounds $n$ arrows directed to $n$ points $x_{1} \ldots x_{n}$ and $\mathrm{n}$ arrows originated from $x_{1}^{\prime} \ldots x_{n}^{\prime}$.

In these diagrams the hopping matrix elements are represented by thin dashed lines, the delocalized electron Green function - by a full solid line. 
In figure 1 the one-particle Green function's diagrams of the first two perturbation theory orders is represented:

The sign of the diagram 1d) corresponds to the enumeration of rectangle arguments $G_{2}^{(0) i r}\left[x 1 \mid 2^{\prime} x^{\prime}\right]$.

We can see from the first three diagrams of figure 1 that the chain iterative process leads to the ordinary Dyson equation with hopping matrix element $t\left(1^{\prime}-1\right)$ as a self-energy.

But new diagrams containing charge and spin fluctuations give rise to an additional renormalization process resulting in the necessity to introduce a new irreducible strongly linked function $Z\left(x \mid x^{\prime}\right)$ in the theory. This function is the sum of all irreducible contributions which cannot be broken into two parts by cutting a single hopping line.

In the second order of the perturbation theory the diagram of figure $1 \mathrm{~d}$ ) is a contribution to this function $Z^{(2)}\left(x \mid x^{\prime}\right)$.

In paper [1] it was proved that by introducing function $\Lambda\left(x \mid x^{\prime}\right) \quad(x=\boldsymbol{x}, \sigma, \tau)$

$$
\Lambda\left(x \mid x^{\prime}\right)=G^{(0)}\left(x \mid x^{\prime}\right)+Z\left(x \mid x^{\prime}\right)
$$

we get the Dyson equation for the renormalized one-particle Green function $G\left(x \mid x^{\prime}\right)$ of the Hubbard model:

$$
G\left(x \mid x^{\prime}\right)=\Lambda\left(x \mid x^{\prime}\right)+\sum_{x_{1}, x_{2}} \Lambda\left(x \mid x_{1}\right) t\left(x_{1}-x_{2}\right) G\left(x_{2} \mid x^{\prime}\right),
$$

where $t\left(x_{1}-x_{2}\right)=t\left(\boldsymbol{x}_{1}-\boldsymbol{x}_{2}\right) \delta_{\sigma_{1} \sigma_{2}} \delta\left(\tau_{1}-\tau_{2}\right)$ and summations stand for summing by discrete indices and integration by imaginary time.

In the Fourier representation we have

$$
G_{\sigma}(\boldsymbol{k} \mid i \omega)=\frac{\Lambda_{\sigma}(\boldsymbol{k} \mid i \omega)}{1-\xi(\boldsymbol{k}) \Lambda_{\sigma}(\boldsymbol{k} \mid i \omega)} .
$$

Here $\boldsymbol{k}$ is the momentum of an electron, $\omega_{n}=(2 n+1) \pi / \beta$ - Matsubara odd frequency and $\xi(k)-$ Fourier component of the hopping matrix element and electron band energy.

Equation (8), in distinction from (7), supposes diagonality by spin indices of all the quantities. If we sum only chain diagrams, without taking into account correlation contributions, we obtain the so-called Hubbard I approximation:

$$
G_{\sigma}^{I}(\boldsymbol{k} \mid i \omega)=\frac{G_{\sigma}^{(0)}(i \omega)}{1-\xi(k) G_{\sigma}^{(0)}(i \omega)},
$$

where $G_{\sigma}^{(0)}(i \omega)$ is the local Hubbard-Green function of an electron. As it is well known, the energy spectrum in this approximation consists of two lower and upper Hubbard subbands.

The complicacy of irreducible contributions to one-particle Green function makes impossible the obtaining of the exact result of a Dyson-type for $Z\left(x \mid x^{\prime}\right)$ function. There are some possibilities to sum a class of irreducible diagrams which contain the most important information about the role of spin and charge fluctuations. 


\section{Ladder diagrams}

The task of this paper is to demonstrate the possibility to sum a special class of irreducible diagrams which take into account two-particle correlation effects.

In figure 2 we demonstrate some diagrams for $Z\left(x \mid x^{\prime}\right)$ function which are essentially for strong correlated systems and will be summed.

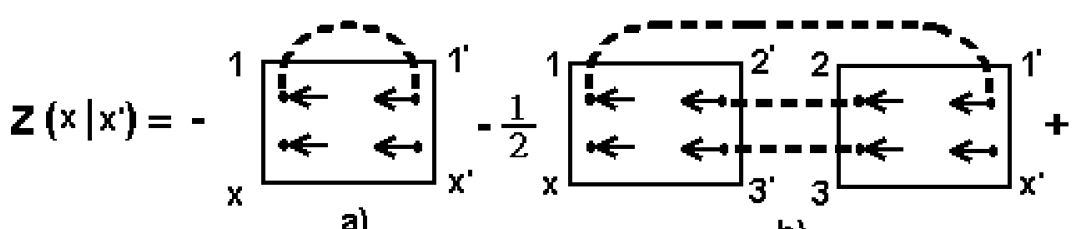

a)

b)

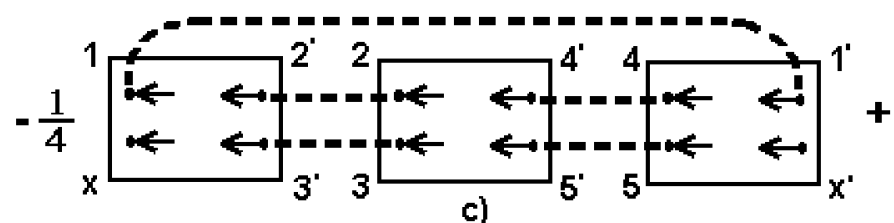

c)

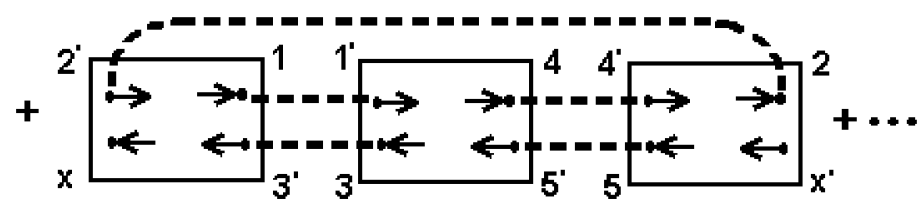

d)

Figure 2. Ladder diagrams for irreducible $Z\left(x \mid x^{\prime}\right)$ function. They repeat the infinite number of times the irreducible two-particle Green functions. The dashed lines represent renormalized hopping matrix elements. The rectangles in these diagrams stand for two-particle irreducible Green functions $G_{2}^{(0) i r}$.

The renormalized hopping matrix element $\widetilde{t}\left(x-x^{\prime}\right)$ is the result of the summing of some elements of diagrams and are determined by the equation:

$$
\widetilde{t}\left(x^{\prime}-x\right)=t\left(x^{\prime}-x\right)+\sum_{x_{1}, x_{2}} t\left(x^{\prime}-x_{1}\right) G\left(x_{1} \mid x_{2}\right) t\left(x_{2}-x\right),
$$

Fourier representation $\xi_{\sigma}(k \mid \omega)$ of quantity $\widetilde{t}\left(x^{\prime}-x\right)$ is equal to

$$
\xi_{\sigma}\left(\boldsymbol{k} \mid i \omega_{n}\right)=\xi(\boldsymbol{k})+\xi^{2}(\boldsymbol{k}) G_{\sigma}\left(\boldsymbol{k} \mid i \omega_{n}\right)=\frac{\xi(\boldsymbol{k})}{1-\xi(\boldsymbol{k}) \Lambda_{\sigma}\left(\boldsymbol{k} \mid i \omega_{n}\right)},
$$

where $\xi(\boldsymbol{k})$ is a tight-binding dispersion law of non-interacting electrons.

For some of the diagrams in figure 2, especially for the diagram in figure 2a, the renormalization process is very important because this diagram with the unrenormalized hopping line is equal to zero, noting that $t\left(x^{\prime}=x\right)=0$. The other diagrams in figure 2 are delocalized $\left(\boldsymbol{x} \neq \boldsymbol{x}^{\prime}\right)$ and their contributions are not zero when single dashed lines are used. 
In figure 2 we have two different kinds of ladder diagrams. One of them represented by diagrams a), b),c) and the next diagrams of the higher order of the perturbation theory are repetitions of the process of scattering two electrons with the conservation of their sum of moments and frequencies.

The second ladder shown by the diagram in figure $2 \mathrm{~d}$ and the next diagrams represent the process of repeated electron-hole scattering with the conservation of their differences of momenta and frequencies.

In the weak coupling theory of the solid state such a summation is known very well and is the realization of the Random Phase Approximation.

The difference between the classical RPA and our ladder generalized RPA consists in the instant in the first case and the retarded in the second case character of electron scattering, which in our case is determined by four imaginary times of irreducible two-particles Green functions represented in the diagrams by the rectangles.

To sum the first class of ladder diagrams of figure 2 for $Z\left(x \mid x^{\prime}\right)$ function we shall investigate a more simple process of delocalization of two-particle irreducible Green functions represented in figure 3. This renormalized function is determined as $G_{2}^{i r}$.

The graphical integral equation (figure $3 \mathrm{~b}$ ) for the delocalized irreducible Green function $G_{2}^{i r}\left[x_{1}, x_{2} \mid x_{2}^{\prime}, x_{1}^{\prime}\right]$ has the form:

$$
\begin{aligned}
& G_{2}^{i r}\left[x_{1}, x_{2} \mid x_{2}^{\prime}, x_{1}^{\prime}\right]=G_{2}^{(0) i r}\left[x_{1}, x_{2} \mid x_{2}^{\prime}, x_{1}^{\prime}\right]+\frac{1}{2} \sum_{11^{\prime} 22^{\prime}} G_{2}^{(0) i r}\left[x_{1}, x_{2} \mid 1^{\prime}, 2^{\prime}\right] \\
& \times \widetilde{t}\left(1^{\prime}-1\right) \widetilde{t}\left(2^{\prime}-2\right) G_{2}^{i r}\left[2,1 \mid x_{2}^{\prime}, x_{1}^{\prime}\right) .
\end{aligned}
$$

Here $x_{i}$ stands, as usual, for $\boldsymbol{x}_{1}, \sigma_{1}, \tau_{1} ; 1-$ for $\mathbf{1}, \alpha_{1}, \theta_{1}$.

The sum $\sum_{1}$ stands for the summing by lattice sites $\mathbf{1}$, spin $\alpha_{1}$ and integration by imaginary time $\theta_{1}$ in the $(0, \beta)$ interval. The site dependence of the renormalized $G_{2}^{i r}$ function is partially diagonal, as it can be seen from figure 3a:

$$
G_{2}^{i r}\left[x_{1}, x_{2} \mid x_{2}^{\prime}, x_{1}^{\prime}\right]=\delta_{\boldsymbol{x}_{1}, \boldsymbol{x}_{2}} \delta_{\boldsymbol{x}_{1}^{\prime}, \boldsymbol{x}_{2}^{\prime}} G_{2}^{i r}\left[\boldsymbol{x}_{1}-\boldsymbol{x}_{1}^{\prime} ; \sigma_{1} \tau_{1} ; \sigma_{2} \tau_{2} \mid \sigma_{2}^{\prime} \tau_{2}^{\prime} ; \sigma_{1}^{\prime} \tau_{1}^{\prime}\right]
$$

The delocalized irreductible Green function, which depends on relative lattice sites, obeys the equation:

$$
\begin{gathered}
G_{2}^{i r}\left[\boldsymbol{x}_{1}-\boldsymbol{x}_{1}^{\prime} ; \sigma_{1} \tau_{1} ; \sigma_{2} \tau_{2} \mid \sigma_{2}^{\prime} \tau_{2}^{\prime} ; \sigma_{1}^{\prime} \tau_{1}^{\prime}\right]=\delta_{\boldsymbol{x}_{1} \boldsymbol{x}_{1}^{\prime}} G_{2}^{(0) i r}\left[\sigma_{1} \tau_{1} ; \sigma_{2} \tau_{2} \mid \sigma_{2}^{\prime} \tau_{2}^{\prime} ; \sigma_{1}^{\prime} \tau_{1}^{\prime}\right]+ \\
+\frac{1}{2} \int_{0}^{\beta} \ldots \int_{0}^{\beta} d \theta_{1} d \theta_{2} d \theta_{1}^{\prime} d \theta_{2}^{\prime} \sum_{\alpha_{1} \alpha_{2}} \sum_{\mathbf{1}} G_{2}^{(0) i r}\left[\sigma_{1} \tau_{1} ; \sigma_{2} \tau_{2} \mid \alpha_{1} \theta_{1}^{\prime}, \alpha_{2} \theta_{2}^{\prime}\right] \times \\
\times \widetilde{t}_{\alpha_{1}}\left(\boldsymbol{x}_{1}-\mathbf{1} \mid \theta_{1}^{\prime}-\theta_{1}\right) t_{\alpha_{2}}\left(\boldsymbol{x}_{1}-\mathbf{1} \mid \theta_{2}^{\prime}-\theta_{2}\right) G_{2}^{i r}\left[\mathbf{1}-\boldsymbol{x}_{1}^{\prime} ; \alpha_{2}, \theta_{2} ; \alpha_{1} \theta_{1} \mid \sigma_{2}^{\prime}, \tau_{2}^{\prime} ; \sigma_{1}^{\prime}, \tau_{1}^{\prime}\right] .
\end{gathered}
$$

To advance the solution of this equation we introduce the momentum and frequency representation of our functions:

$$
G_{2}\left[\sigma_{1} \tau_{1} ; \sigma_{2} \tau_{2} \mid \sigma_{2}^{\prime} \tau_{2}^{\prime} ; \sigma_{1}^{\prime} \tau_{1}^{\prime}\right]=
$$




$$
\begin{gathered}
=\frac{1}{\beta^{4}} \sum_{\omega_{1} \omega_{2} \omega_{2^{\prime}} \omega_{1^{\prime}}} G_{2}\left[\sigma_{1}, i \omega_{1} ; \sigma_{2}, i \omega_{2} \mid \sigma_{2}^{\prime}, i \omega_{2}^{\prime} ; \sigma, i \omega_{1}^{\prime}\right] e^{-i \omega_{1} \tau_{1}-i \omega_{2} \tau_{2}+i \omega_{2}^{\prime} \tau_{2}^{\prime}+i \omega_{1}^{\prime} \tau_{1}^{\prime}}, \\
\left.Z\left(x \mid x^{\prime}\right)=\frac{1}{\beta N} \sum_{\boldsymbol{k}} \sum_{\omega} Z_{\sigma \sigma^{1}}(\boldsymbol{k} \mid i \omega) e^{-i \boldsymbol{k}\left(\boldsymbol{x}-\boldsymbol{x}^{\prime}\right)-i \omega\left(\tau-\tau^{\prime}\right)}\right) .
\end{gathered}
$$

That permits us to rewrite equation (14) in the form:

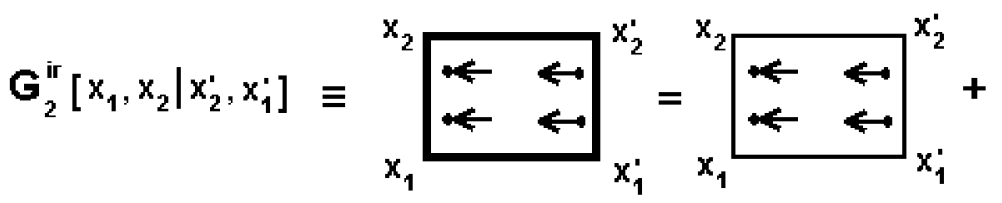

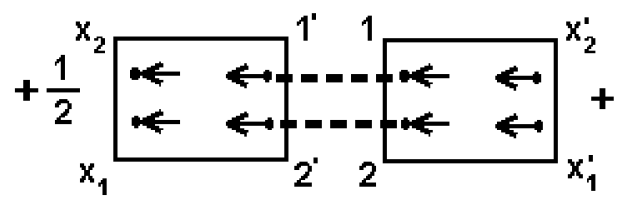

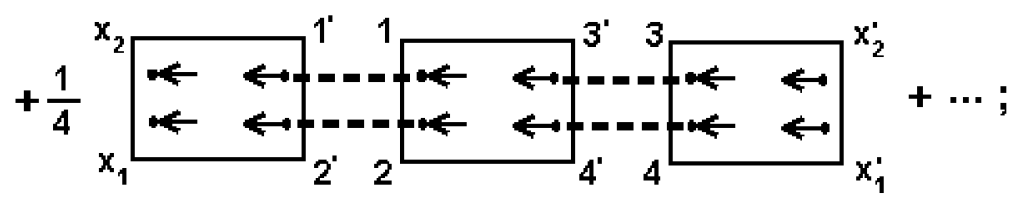

a)
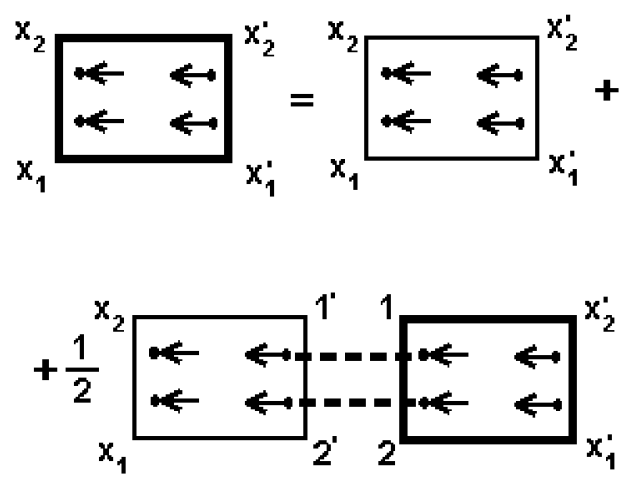

b)

Figure 3. Ladder diagrams (figure 3a) for the delocalized two-particle irreducible Green function $G_{2}^{i r}$ and the graphical integral equation (figure $3 \mathrm{~b}$ ) for their summing. The rectangles with full lines stand for delocalized and with thin lines for localized irreducible functions.

$$
\begin{gathered}
G_{2, \boldsymbol{q}}^{i r}\left[\sigma_{1}, i \omega_{1} ; \sigma_{2}, i \omega_{2} \mid \sigma_{2}^{\prime}, i \omega_{2}^{\prime} ; \sigma_{1}^{\prime}, i \omega_{1}^{\prime}\right]=G_{2}^{(0) i r}\left[\sigma_{1}, i \omega_{1} ; \sigma_{2}, i \omega_{2} \mid \sigma_{2}^{\prime}, i \omega_{2}^{\prime} ; \sigma_{1}^{\prime}, i \omega_{1}^{\prime}\right]+ \\
+\frac{1}{2 \beta^{2}} \sum_{\alpha_{1} \alpha_{2}} \sum_{\omega_{1}^{\prime \prime}, \omega_{2}^{\prime \prime}} G_{2}^{(0) i r}\left[\sigma_{1}, i \omega_{1} ; \sigma_{2}, i \omega_{2} \mid \alpha_{1}, i \omega_{1}^{\prime \prime} ; \alpha_{2}, i \omega_{2}^{\prime \prime}\right] \times
\end{gathered}
$$




$$
\times \xi_{2, \boldsymbol{q}}\left[\alpha_{1}, i \omega_{1}^{\prime \prime} ; \alpha_{2}, i \omega_{2}^{\prime \prime}\right] G_{2, \boldsymbol{q}}^{i r}\left[\alpha_{2}, i \omega_{2}^{\prime \prime} ; \alpha_{1}, i \omega_{1}^{\prime \prime} \mid \sigma_{2}^{\prime}, i \omega_{2}^{\prime} ; \sigma_{1}^{\prime}, i \omega_{1}^{\prime}\right]
$$

where

$$
\xi_{2, \boldsymbol{q}}\left[\alpha_{1}, i \omega_{1}^{\prime \prime}, \alpha_{2}, i \omega_{2}^{\prime \prime}\right]=\frac{1}{N} \sum_{\boldsymbol{q}_{1}} \xi_{\alpha_{1}}\left(\boldsymbol{q}_{1} \mid i \omega_{1}^{\prime \prime}\right) \xi_{\alpha_{2}}\left(\boldsymbol{q}-\boldsymbol{q}_{1} \mid i \omega_{2}^{\prime \prime}\right) .
$$

All the irreducible functions demonstrate spin and frequency conservation according to the fact that the total spin and frequency of the annihilated electrons are equal to those of the created electrons:

$$
\begin{gathered}
G_{2}^{(0) i r}\left[\sigma_{1}, i \omega_{1} ; \sigma_{2}, i \omega_{2} \mid \sigma_{3}, i \omega_{3} ; \sigma_{4}, i \omega_{4}\right]=\beta \delta\left(\omega_{1}+\omega_{2}-\omega_{3}-\omega_{4}\right) \times \\
\widetilde{G}_{2}^{(0) i r}\left[\sigma_{1}, i \omega_{1} ; \sigma_{2}, i \omega_{2} \mid \sigma_{3}, i \omega_{3} ; \sigma_{4}, \omega_{4}\right]=\delta_{\sigma_{1} \sigma_{3}} \delta_{\sigma_{2} \sigma_{4}}\left[K_{1}\left[\sigma_{1}, i \omega_{1} ; \sigma_{2}, i \omega_{2} \mid \sigma_{1}, i \omega_{3} ; \sigma_{2}, i \omega_{4}\right]+\right. \\
\left.\beta^{2} \delta\left(\omega_{1}+\omega_{2}-\omega_{3}-\omega_{4}\right) \delta\left(\omega_{1}-\omega_{3}\right) \widetilde{G}_{\sigma_{1}}^{(0)}\left(i \omega_{1}\right) \widetilde{G}_{\sigma_{2}}^{(0)}\left(i \omega_{2}\right)\right]+\delta_{\sigma_{1} \sigma_{4}} \delta_{\sigma_{2} \sigma_{3}} \times \\
{\left[K_{2}\left[\sigma_{1} i \omega_{1} ; \sigma_{2} i \omega_{2} \mid \sigma_{2} i \omega_{3} ; \sigma_{1} i \omega_{4}\right]-\beta^{2} \delta\left(\omega_{1}+\omega_{2}-\omega_{3}-\omega_{4}\right) \delta\left(\omega_{1}-\omega_{4}\right) \times\right.} \\
\left.\widetilde{G}_{\sigma_{1}}^{(0)}\left(i \omega_{1}\right) \widetilde{G}_{\sigma_{2}}^{(0)}\left(i \omega_{2}\right)\right]-\sigma_{1} \sigma_{3} \delta_{\sigma_{2},-\sigma_{1}} \delta_{\sigma_{4}-\sigma_{3}} K_{3}\left[\sigma_{1}, i \omega_{1} ;-\sigma_{1}, i \omega_{2} \mid \sigma_{3}, i \omega_{3} ;-\sigma_{3}, i \omega_{4}\right] .
\end{gathered}
$$

The functions $K_{i}, i=1,2,3$, are the contributions of different spin channels from the two-particle on-site Green function, while the bilinear on $\widetilde{G}_{\sigma}^{(0)}(i \omega)$ terms are the substracted from Kubo cumulant members. All the $K_{i}$ functions are proportional to the factor $\beta \delta\left(\omega_{1}+\omega_{2}-\omega_{3}-\omega_{4}\right)$ which expresses the frequencies conservation law. Some values of these $K_{i}$ functions may be found in [1]. These laws of conservation are also valid for the renormalized $G_{2}^{i r}$ quantity.

To take into account the frequency conservation law we go from $G^{i r}$ to $\widetilde{G}^{i r}$ functions and define new frequencies using the even quantity $\Omega=2 m \pi / \beta$

$$
\begin{aligned}
& \widetilde{G}_{2, \boldsymbol{q}}^{i r}\left[\sigma, i(\omega+\Omega) ; \sigma_{1}, i\left(\omega_{1}-\Omega_{1}\right) \mid \sigma_{2}, i \omega_{1} ; \sigma^{\prime}, i \omega\right]= \\
& =\widetilde{G}_{2}^{(0) i r}\left[\sigma, i(\omega+\Omega) ; \sigma_{1}, i\left(\omega_{1}-\Omega\right) \mid \sigma_{2}, i \omega_{1} ; \sigma^{\prime}, i \omega\right]+ \\
& +\frac{1}{2 \beta} \sum_{\Omega_{1} \alpha_{1} \alpha_{2}} \widetilde{G}^{(0) i r}\left[\sigma, i(\omega+\Omega) ; \sigma_{1}, i\left(\omega_{1}-\Omega\right) \mid \alpha_{1}, i\left(\omega_{1}-\Omega_{1}\right) ; \alpha_{2}, i\left(\omega+\Omega_{1}\right)\right] \\
& \times \xi_{2, \boldsymbol{q}}\left[\alpha_{1}, i\left(\omega_{1}-\Omega_{1}\right) ; \alpha_{2}, i\left(\omega+\Omega_{1}\right)\right] \\
& \times \widetilde{G}_{2, \boldsymbol{q}}^{i r}\left[\alpha_{2}, i\left(\omega+\Omega_{1}\right) ; \alpha_{1},\left(\omega_{1}-\Omega_{1}\right) \mid \sigma_{2}, i \omega_{1} ; \sigma^{\prime}, i \omega\right] .
\end{aligned}
$$

Using the definition of renormalized $G_{2}^{i r}$ function we can write down the contribution of the first class of the ladder diagrams in figure 2 to the function $Z\left(x \mid x^{\prime}\right)$ in the form:

$$
Z^{I}\left(x \mid x^{\prime}\right)=-\sum_{x_{1} x_{1}^{\prime}} G_{2}^{i r}\left[x, x_{1} \mid x_{1}^{\prime}, x^{\prime}\right] \widetilde{t}\left(x_{1}^{\prime}-x_{1}\right)
$$

On the basis of (13) and (18) this equation may be rewritten as

$$
\begin{gathered}
Z_{\sigma \sigma^{\prime}}^{I}\left(\boldsymbol{x}-\boldsymbol{x}^{\prime} \mid \tau-\tau^{\prime}\right)=-\sum_{\sigma_{1}, \sigma_{1}^{\prime}} \iint d \tau_{1} d \tau_{1}^{\prime} G_{2}^{i r}\left[\boldsymbol{x}-\boldsymbol{x}^{\prime} ; \sigma \tau, \sigma_{1} \tau_{1} \mid \sigma_{1}^{\prime}, \tau_{1}^{\prime} ; \sigma^{\prime} \tau^{\prime}\right] \\
\times \widetilde{t}_{\sigma_{1}^{\prime} \sigma_{1}}\left(\boldsymbol{x}^{\prime}-\boldsymbol{x}, \tau_{1}^{\prime}-\tau_{1}\right)=-\frac{1}{\beta^{3}} \sum_{\sigma_{1}, \sigma_{1}^{\prime}, \omega, \omega^{\prime}, \omega_{1}} \widetilde{t}_{\sigma_{1}^{\prime} \sigma_{1}}\left(\boldsymbol{x}^{\prime}-\boldsymbol{x} ; i \omega_{1}\right) \\
\times G_{2}^{i r}\left[\boldsymbol{x}-\boldsymbol{x}^{\prime}, \sigma, i \omega ; \sigma_{1}, i \omega_{1} \mid \sigma_{1}^{\prime}, i \omega_{1} ; \sigma^{\prime}, i \omega\right] e^{-i \omega \tau+i \omega^{\prime} \tau^{\prime}}
\end{gathered}
$$


or

$$
Z_{\sigma}^{I}(\boldsymbol{q} \mid i \omega)=-\frac{1}{N} \frac{1}{\beta} \sum_{\boldsymbol{k}_{1} \omega_{1} \sigma_{1}} \widetilde{G}_{2, \boldsymbol{k}_{1}}^{i r}\left[\sigma, i \omega ; \sigma_{1}, i \omega_{1} \mid \sigma_{1}, i \omega_{1}, \sigma, i \omega\right] * \xi_{\sigma_{1}}\left(\boldsymbol{k}_{1}-\boldsymbol{q} \mid i \omega_{1}\right)
$$

According to equation (21), to find $Z^{I}$ we need a more simple modification of renormalized $G_{2}^{i r}$ function with equality in pairs of frequencies and spins. But it is necessary, at the beginning, to find this function for a more general case and then to put a specific condition $\Omega=0$ in it.

Now we take into account that the spin of the propagating electron is conserved $\sigma=\sigma^{\prime}$ (no magnetic fields and magnetic structures), and so is the conservation law of the spin in all the intermediate scattering processes. Then we have $\sigma_{2}=\sigma_{1}$ and $\sigma+\sigma_{1}=\alpha_{1}+\alpha_{2}$. Because $\sigma_{1}$ can be equal to $\pm \sigma$, we shall consider these two possibilities separately. In the first case $\sigma_{1}=\sigma$ we have $\alpha_{1}=\alpha_{2}=\sigma$ and equation (19) in a more simple form:

$$
\begin{gathered}
\left.\widetilde{G}_{2, \boldsymbol{q}}^{i r}\left[\sigma, i(\omega+\Omega), \sigma, i\left(\omega_{1}-\Omega\right) \mid \sigma, i \omega_{1} ; \sigma, i \omega\right)\right]= \\
=\widetilde{G}_{2}^{(0) i r}\left[\sigma, i(\omega+\Omega) ; \sigma, i\left(\omega_{1}-\Omega\right) \mid \sigma, i \omega_{1}, \sigma, i \omega\right] \\
+\frac{1}{2 \beta} \sum_{\Omega_{1}} \widetilde{G}_{2}^{(0) i r}\left[\sigma, i(\omega+\Omega) ; \sigma, i\left(\omega_{1}-\Omega\right) \mid \sigma, i\left(\omega_{1}-\Omega_{1}\right), \sigma, i\left(\omega+\Omega_{1}\right)\right] \\
\times \xi_{2, \boldsymbol{q}}\left[\sigma, i\left(\omega_{1}-\Omega_{1}\right) ; \sigma, i\left(\omega+\Omega_{1}\right)\right] \\
\times \widetilde{G}_{2, \boldsymbol{q}}^{i r}\left[\sigma, i\left(\omega+\Omega_{1}\right) ; \sigma, i\left(\omega_{1}-\Omega_{1}\right) \mid \sigma, i \omega_{1} ; \sigma, i \omega\right] .
\end{gathered}
$$

But in the second case $\sigma_{1}=-\sigma$,we have two possibilities to select $\alpha_{1}=-\alpha_{2}= \pm \sigma$ and equation (19) takes the form:

$$
\begin{gathered}
\widetilde{G}_{2, \boldsymbol{q}}^{i r}\left[\sigma, i(\omega+\Omega) ;-\sigma, i\left(\omega_{1}-\Omega\right) \mid-\sigma, i \omega_{1} ; \sigma, i \omega\right]= \\
=\widetilde{G}_{2}^{(0) i r}\left[\sigma, i(\omega+\Omega),-\sigma, i\left(\omega_{1}-\Omega\right) \mid-\sigma, i \omega_{1} ; \sigma, i \omega\right]+ \\
+\frac{1}{2 \beta} \sum_{\Omega_{1}} \widetilde{G}_{2}^{(0) i r}\left[\sigma, i(\omega+\Omega) ;-\sigma, i\left(\omega_{1}-\Omega\right) \mid \sigma, i\left(\omega_{1}-\Omega_{1}\right) ;-\sigma, i\left(\omega+\Omega_{1}\right)\right] \\
\times \xi_{2, \boldsymbol{q}}\left[\sigma, i\left(\omega_{1}-\Omega_{1}\right),-\sigma, i\left(\omega+\Omega_{1}\right)\right] \\
\times \widetilde{G}_{2, \boldsymbol{q}}^{i r}\left[-\sigma, i\left(\omega+\Omega_{1}\right) ; \sigma, i\left(\omega_{1}-\Omega_{1}\right) \mid-\sigma, i \omega_{1} ; \sigma, i \omega\right]+ \\
+\frac{1}{2 \beta} \sum_{\Omega_{1}} \widetilde{G}_{2}^{(0) i r}\left[\sigma, i(\omega+\Omega) ;-\sigma, i\left(\omega_{1}-\Omega\right) \mid-\sigma, i\left(\omega_{1}-\Omega_{1}\right) ; \sigma, i\left(\omega+\Omega_{1}\right)\right] \\
\times \xi_{2, \boldsymbol{q}}\left[-\sigma, i\left(\omega_{1}-\Omega_{1}\right), \sigma, i\left(\omega+\Omega_{1}\right)\right] \\
\times \widetilde{G}_{2, \boldsymbol{q}}^{i r}\left[\sigma, i\left(\omega+\Omega_{1}\right) ;-\sigma, i\left(\omega_{1}-\Omega_{1}\right) \mid-\sigma, i \omega_{1} ; \sigma, i \omega\right] .
\end{gathered}
$$

In the second term of the right-hand side of equation (23) we can make the following substitution of $\Omega_{1}$ by variable $\Omega_{1}=\omega_{1}-\omega-\Omega_{2}^{\prime}$ and take into account the antisymmetric properties of local and delocalized irreducible Green functions according to the permutation of their arguments. The result is the equality of the last two terms of (23). We can use only one of them multiplying by the coefficient 
two. We have

$$
\begin{gathered}
\widetilde{G}_{2, \boldsymbol{q}}^{i r}\left[\sigma, i(\omega+\Omega) ;-\sigma, i\left(\omega_{1}-\Omega\right) \mid-\sigma, i \omega_{1} ; \sigma, i \omega\right]= \\
=\widetilde{G}_{2}^{(0) i r}\left[\sigma, i(\omega+\Omega) ;-\sigma, i\left(\omega_{1}-\Omega\right) \mid-\sigma, i \omega_{1} ; \sigma, i \omega\right]+ \\
+\frac{1}{\beta} \sum_{\Omega_{1}} \widetilde{G}_{2}^{(0) i r}\left[\sigma, i(\omega+\Omega) ;-\sigma, i\left(\omega_{1}-\Omega\right) \mid-\sigma, i\left(\omega_{1}-\Omega_{1}\right) ; \sigma, i\left(\omega+\Omega_{1}\right)\right] \\
\times \xi_{2, \boldsymbol{q}}\left[-\sigma, i\left(\omega_{1}-\Omega_{1}\right) ; \sigma, i\left(\omega+\Omega_{1}\right)\right] \\
\left.\times \widetilde{G}_{2, \boldsymbol{q}}^{i r}\left[\sigma, i\left(\omega+\Omega_{1}\right) ;-\sigma, i\left(\omega_{1}-\Omega_{1}\right) \mid-\sigma, i \omega_{1} ; \sigma, i \omega\right)\right] .
\end{gathered}
$$

To solve equations (22) and (24) it is necessary to know two kernels of these integral equations, namely, $\widetilde{G}_{2}^{(0) i r}\left[\sigma, i(\omega+\Omega) ; \sigma, i\left(\omega_{1}-\Omega\right) \mid \sigma, i\left(\omega_{1}-\Omega_{1}\right) ; \sigma, i\left(\omega+\Omega_{1}\right)\right]$ and $\widetilde{G}_{2}^{(0) i r}\left[\sigma, i(\omega+\Omega),-\sigma, i\left(\omega_{1}-\Omega\right) \mid-\sigma i\left(\omega_{1}-\Omega\right), \sigma i\left(\omega+\Omega_{1}\right)\right]$ which were studied in papers [1-2] and will be discussed below.

To find the second contribution to the irreducible function $Z\left(x \mid x^{\prime}\right)$ we have to analyse diagrams d) in figure 2 and the analogous diagrams of the higher order of the perturbation theory (see figure 4). The infinite series of such kind of diagrams belong to the new renormalized and delocalized function $H_{2}^{i r}\left[x_{1} x_{2} \mid x_{2} x_{1}^{\prime}\right]$.

All such diagrams have a coefficient equal to one. The sign of the diagrams corresponds to a special order of arguments of all the irreducible functions. It must be of a clockwise order if we begin to count from the lower left corner of the rectangle or counter-clockwise if the count begins from the lower right corner of the rectangle. The function $H_{2}^{i r}$ has partial local properties

$$
H_{2}^{i r}\left[x_{1}, x_{2}^{\prime} \mid x_{2}, x_{1}^{\prime}\right]=\delta_{\boldsymbol{x}_{1,} \boldsymbol{x}_{2}^{\prime}} \delta_{\boldsymbol{x}_{2}, \boldsymbol{x}_{1}^{\prime}} H_{2}^{i r}\left[\boldsymbol{x}_{1}-\boldsymbol{x}_{1}^{\prime} ; \sigma_{1} \tau_{1} ; \sigma_{2}^{\prime}, \tau_{2} \mid \sigma_{2}, \tau_{2}^{\prime} ; \sigma_{1}^{\prime}, \tau_{1}^{\prime}\right] .
$$

This new function, which depends on the relative arguments $\boldsymbol{x}_{1}-\boldsymbol{x}_{1}^{\prime}$, obeys the integral equation (see figure $4 \mathrm{~b}$ ):

$$
\begin{gathered}
H_{2}^{i r}\left[\boldsymbol{x}_{1}-\boldsymbol{x}_{1}^{\prime} ; \sigma_{1}, \tau_{1} ; \sigma_{2}^{\prime}, \tau_{2}^{\prime} \mid \sigma_{2}, \tau_{2} ; \sigma_{1}^{\prime} \tau_{1}^{\prime}\right]=-\delta_{\boldsymbol{x}_{1}, \boldsymbol{x}_{1}^{\prime}} G_{2}^{(0) i r}\left[\sigma_{1}, \tau_{1} ; \sigma_{2}, \tau_{2} \mid \sigma_{2}^{\prime}, \tau_{2}^{\prime} ; \sigma_{1}^{\prime}, \tau_{1}^{\prime}\right]+ \\
+\sum_{1} \sum_{\alpha_{1}, \alpha_{2}} \int . \iint d \theta_{1} d \theta_{2} d \theta_{1}^{\prime} d \theta_{2}^{\prime} G_{2}^{(0) i r}\left[\sigma_{1}, \tau_{1} ; \alpha_{1} \theta_{1} \mid \sigma_{2}^{\prime} \tau_{2}^{\prime} \alpha_{2} \theta_{2}^{\prime}\right] \\
\times \widetilde{t}_{\alpha_{1}}\left(\mathbf{1}-\boldsymbol{x}_{1} \mid \theta_{1}^{\prime}-\theta_{1}\right) \widetilde{t}_{\alpha_{2}}\left(\boldsymbol{x}_{1}-\mathbf{1} \mid \theta_{2}^{\prime}-\theta_{2}\right) \\
\times H_{2}^{i r}\left[\mathbf{1}-\boldsymbol{x}_{1}^{\prime} ; \alpha_{2}, \theta_{2} ; \alpha_{1}, \theta_{1}^{\prime} \mid \sigma_{2}, \tau_{2} ; \sigma_{1}^{\prime}, \tau_{1}^{\prime}\right]
\end{gathered}
$$

Using the Fourier representation we obtain

$$
\begin{aligned}
& H_{2, \boldsymbol{q}}^{i r}\left[\sigma_{1}, i \omega_{1} ; \sigma_{2}^{\prime}, i \omega_{2}^{\prime} \mid \sigma_{2}, i \omega_{2} ; \sigma_{1}^{\prime}, \omega_{1}^{\prime}\right]= \\
& -G_{2}^{(0) i r}\left[\sigma_{1}, i \omega_{1} ; \sigma_{2}, i \omega_{2} \mid \sigma_{2}^{\prime}, i \omega_{2}^{\prime} ; \sigma_{1}^{\prime}, i \omega_{1}^{\prime}\right]+ \\
& +\frac{1}{\beta^{2}} \sum_{\omega_{1}^{\prime \prime}} \sum_{\omega_{2}^{\prime \prime}} \sum_{\alpha_{1} \alpha_{2}} \xi_{2, \boldsymbol{q}}\left[\alpha_{1}, i \omega_{1}^{\prime \prime} ; \alpha_{2}, i \omega_{2}^{\prime \prime}\right] G_{2}^{(0) i r}\left[\sigma_{1}, i \omega_{1} ; \alpha_{1}, i \omega_{1}^{\prime \prime} \mid \sigma_{2}^{\prime}, i \omega_{2}^{\prime} ; \alpha_{2}, i \omega_{2}^{\prime \prime}\right] \\
& \times H_{2, \boldsymbol{q}}^{i r}\left[\alpha_{2}, i \omega_{2}^{\prime \prime} ; \alpha_{1}, i \omega_{1}^{\prime \prime} \mid \sigma_{2}, i \omega_{2} ; \sigma_{1}^{\prime}, i \omega_{1}^{\prime}\right] .
\end{aligned}
$$

Here we suppose diagonality by spin indices of the renormalized hopping elements and an even symmetry of tight-binding electron energy $\xi(\boldsymbol{k})=\xi(-\boldsymbol{k})$. 


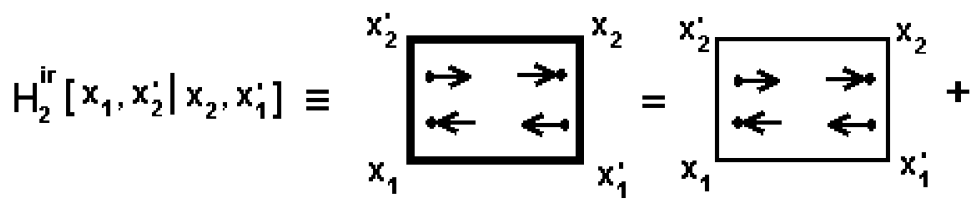
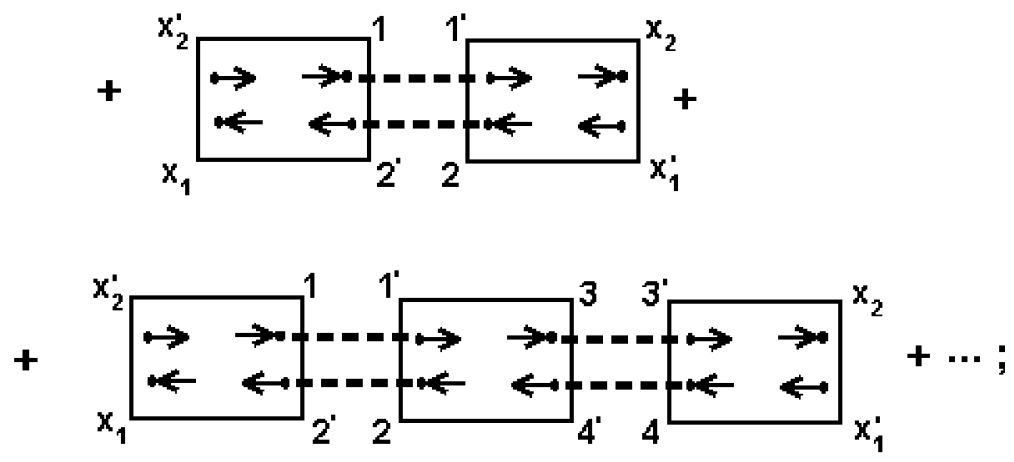

a)
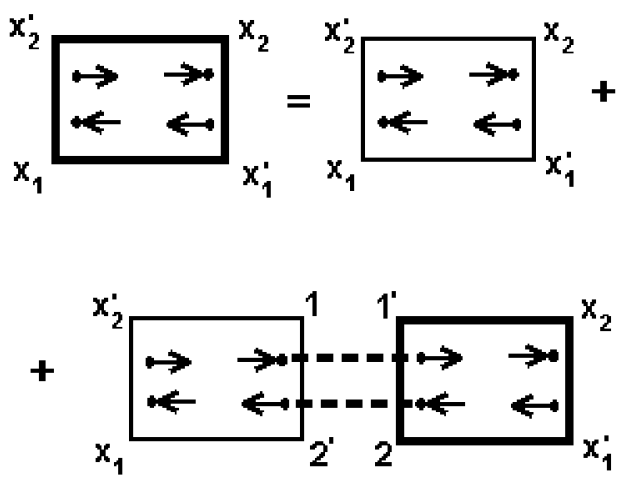

b)

Figure 4. The summation of the second class of ladder diagrams. a) Infinite series of diagrams for the renormalized $H_{2}^{i r}$ function. b) Graphical integral equation for the renormalized $H_{2}^{i r}$ function.

Now we shall take into account the law of frequency conservation both for the local and delocalized irreducible functions. The latter have the property

$$
\begin{gathered}
H_{2, \boldsymbol{q}}^{i r}\left[\sigma_{1}, i \omega_{1} ; \sigma_{2}^{\prime}, i \omega_{2}^{\prime} \mid \sigma_{2}, i \omega_{2} ; \sigma_{1}^{\prime}, i \omega_{1}^{\prime}\right]=\beta \delta\left(\omega_{1}+\omega_{2}-\omega_{2}^{\prime}-\omega_{1}^{\prime}\right) \\
\times \widetilde{H}_{2, \boldsymbol{q}}^{i r}\left[\sigma_{1}, i \omega_{1} ; \sigma_{2}^{\prime}, i \omega_{2}^{\prime} \mid \sigma_{2}, i \omega_{2} ; \sigma_{1}^{\prime} i\left(\omega_{1}+\omega_{2}-\omega_{2}^{\prime}\right] .\right.
\end{gathered}
$$

Then we use in (27) and (28) new frequencies which obey the law:

$$
\begin{gathered}
\omega_{1}^{\prime \prime}=\omega_{2}^{\prime}+\Omega_{1}, \omega_{2}^{\prime \prime}=\omega_{1}+\Omega_{1}, \\
\omega_{2}^{\prime}=\omega_{2}+\Omega, \omega_{1}^{\prime}=\omega_{1}-\Omega .
\end{gathered}
$$


Here $\Omega$ and $\Omega_{1}$ are even Matsubara frequencies. On the basis of equations (27)-(29) we obtain:

$$
\begin{gathered}
\widetilde{H}_{2, \boldsymbol{q}}^{i r}\left[\sigma, i \omega ; \sigma_{1}^{\prime}, i\left(\omega_{1}+\Omega\right) \mid \sigma_{1}, i \omega_{1} ; \sigma^{\prime}, i(\omega-\Omega)\right]= \\
-\widetilde{G}_{2}^{(0) i r}\left[\sigma, i \omega ; \sigma_{1}, i \omega_{1} \mid \sigma_{1}^{\prime}, i\left(\omega_{1}+\Omega\right), \sigma^{\prime}, i(\omega-\Omega)\right]+ \\
+\frac{1}{\beta} \sum_{\Omega_{1}, \alpha_{1}, \alpha_{2}} \widetilde{G}_{2}^{(0) i r}\left[\sigma, i \omega ; \alpha_{1}, i\left(\omega_{1}+\Omega_{1}+\Omega\right) \mid \sigma_{1}^{\prime}, i\left(\omega_{1}+\Omega\right) ; \alpha_{2}, i\left(\omega+\Omega_{1}\right)\right] \\
\times \xi_{2,-\boldsymbol{q}}\left[\alpha_{1}, i\left(\omega_{1}+\Omega_{1}+\Omega\right) ; \alpha_{2}, i\left(\omega+\Omega_{1}\right)\right] \\
\times \widetilde{H}_{2 q}^{i r}\left[\alpha_{2}, i\left(\omega+\Omega_{1}\right), \alpha_{1}, i\left(\omega+\Omega_{1}+\Omega\right) \mid \sigma_{1}, i \omega_{1} ; \sigma^{\prime}, i(\omega-\Omega)\right] .
\end{gathered}
$$

The law of spin conservation is fulfilled here in the form $\sigma+\sigma_{1}=\sigma^{\prime}+\sigma_{1}^{\prime}$ and $\sigma+\alpha_{1}=\sigma_{1}^{\prime}+\alpha_{2}$. If we are interested in the case $\sigma^{\prime}=\sigma$, then we have to discuss only the equalities $\sigma_{1}^{\prime}=\sigma_{1}$ and $\sigma+\alpha_{1}=\sigma_{1}+\alpha_{2}$. As a consequence, when $\sigma_{1}=\sigma$ we have $\alpha_{1}=\alpha_{2}= \pm \sigma$, but when $\sigma_{1}=-\sigma$ we have $\alpha_{1}=-\alpha_{2}=-\sigma$.

The corresponding integral equations are the following:

$$
\begin{gathered}
\widetilde{H}_{2, \boldsymbol{q}}^{i r}\left[\sigma, i \omega ;-\sigma, i\left(\omega_{1}+\Omega\right) \mid-\sigma, i \omega_{1} ; \sigma, i(\omega-\Omega)\right]= \\
-\widetilde{G}_{2}^{(0) i r}\left[\sigma, i \omega ;-\sigma, i \omega_{1} \mid-\sigma, i\left(\omega_{1}+\Omega\right) ; \sigma, i(\omega-\Omega)\right]+ \\
+\frac{1}{\beta} \sum_{\Omega_{1}} \widetilde{G}_{2}^{(0) i r}\left[\sigma, i \omega ;-\sigma, i\left(\omega_{1}-\Omega_{1}+\Omega\right) \mid-\sigma, i\left(\omega_{1}+\Omega\right) ; \sigma, i\left(\omega-\Omega_{1}\right)\right] \\
\times \xi_{2-q}\left[-\sigma, i\left(\omega_{1}-\Omega_{1}+\Omega\right) ; \sigma, i\left(\omega-\Omega_{1}\right)\right] \\
\times \widetilde{H}_{2, \boldsymbol{q}}^{i r}\left[\sigma, i\left(\omega-\Omega_{1}\right) ;-\sigma, i\left(\omega_{1}-\Omega_{1}+\Omega\right) \mid-\sigma, i \omega_{1} ; \sigma, i(\omega-\Omega)\right]
\end{gathered}
$$

and

$$
\begin{gathered}
\widetilde{H}_{2, \boldsymbol{q}}^{i r}\left[\sigma, i \omega ; \sigma, i\left(\omega_{1}+\Omega\right) \mid \sigma, i \omega_{1} ; \sigma, i(\omega-\Omega)\right]= \\
-\widetilde{G}_{2}^{(0) i r}\left[\sigma, i \omega ; \sigma, i \omega_{1} ; \mid \sigma, i\left(\omega_{1}+\Omega\right), \sigma, i(\omega-\Omega)\right]+ \\
+\frac{1}{\beta} \sum_{\Omega_{1}} \widetilde{G}_{2}^{(0) i r}\left[\sigma, i \omega ; \sigma, i\left(\omega_{1}+\Omega_{1}+\Omega\right) \mid \sigma, i\left(\omega_{1}+\Omega\right) ; \sigma, i\left(\omega+\Omega_{1}\right)\right] \\
\times \xi_{2,-\boldsymbol{q}}\left[\sigma, i\left(\omega_{1}+\Omega_{1}+\Omega\right) ; \sigma, i\left(\omega+\Omega_{1}\right)\right] \\
\times \widetilde{H}_{2, \boldsymbol{q}}^{i r}\left[\sigma, i\left(\omega+\Omega_{1}\right), \sigma, i\left(\omega_{1}+\Omega_{1}+\Omega\right) \mid \sigma, i \omega_{1}, \sigma, i(\omega-\Omega)\right]+ \\
+\frac{1}{\beta} \sum_{\Omega_{1}} \widetilde{G}_{2}^{(0) i r}\left[\sigma, i \omega ;-\sigma, i\left(\omega_{1}+\Omega_{1}+\Omega\right) \mid \sigma, i\left(\omega_{1}+\Omega\right) ;-\sigma, i\left(\omega+\Omega_{1}\right)\right] \\
\times \widetilde{H}_{2, \boldsymbol{q}}^{i r}\left[-\sigma, i\left(\omega+\Omega_{1}\right) ;-\sigma, i\left(\omega_{1}+\Omega_{1}+\Omega\right) \mid \sigma, i \omega_{1} ; \sigma, i(\omega-\Omega)\right] .
\end{gathered}
$$

In distinction from (31) equation (32) is not closed because a new function with quite different spin indices appears in the right-hand side of it. 
Returning to equation (30), we can find the lacking equation:

$$
\begin{gathered}
\widetilde{H}_{2, \boldsymbol{q}}^{i r}\left[-\sigma, i \omega ;-\sigma, i\left(\omega_{1}+\Omega\right) \mid \sigma, i \omega_{1} ; \sigma, i(\omega-\Omega)\right]= \\
-\widetilde{G}_{2}^{(0) i r}\left[-\sigma, i \omega ; \sigma i \omega_{1} \mid-\sigma, i\left(\omega_{1}+\Omega\right) ; \sigma, i(\omega-\Omega)\right] \\
-\frac{1}{\beta} \sum_{\Omega_{1}} \widetilde{G}_{2}^{(0) i r}\left[-\sigma, i \omega ; \sigma, i\left(\omega_{1}+\Omega_{1}+\Omega\right) \mid-\sigma, i\left(\omega_{1}+\Omega\right) ; \sigma, i\left(\omega+\Omega_{1}\right)\right] \\
\times \xi_{2,-\boldsymbol{q}}\left[\sigma, i\left(\omega_{1}+\Omega_{1}+\Omega\right) ; \sigma, i\left(\omega+\Omega_{1}\right)\right] \\
\times \widetilde{H}_{2, \boldsymbol{q}}^{i r}\left[\sigma, i\left(\omega+\Omega_{1}\right) ; \sigma, i\left(\omega_{1}+\Omega_{1}+\Omega\right) \mid \sigma, i \omega_{1} ; \sigma, i(\omega-\Omega)\right] \\
-\frac{1}{\beta} \sum_{\Omega_{1}} \widetilde{G}_{2}^{(0) i r}\left[-\sigma, i \omega ;-\sigma, i\left(\omega_{1}+\Omega_{1}+\Omega\right) \mid-\sigma, i\left(\omega_{1}+\Omega\right),-\sigma, i\left(\omega+\Omega_{1}\right)\right] \\
\times \xi_{2,-\boldsymbol{q}}\left[-\sigma, i\left(\omega_{1}+\Omega_{1}+\Omega\right) ;-\sigma, i\left(\omega+\Omega_{1}\right)\right] \\
\times \widetilde{H}_{2, \boldsymbol{q}}^{i r}\left[-\sigma, i\left(\omega+\Omega_{1}\right) ;-\sigma, i\left(\omega_{1}+\Omega_{1}+\Omega\right) \mid \sigma, i \omega_{1} ; \sigma, i(\omega-\Omega)\right] .
\end{gathered}
$$

Equation (32) and (33) have to be solved together, while (31) - separately. To do that, it is necessary to know the kernels of these equations which are localized irreducible two-particle Green functions.

In a special case of half-filling, when the number of electrons is equal to the lattice sites number, the chemical potential of the system $\mu$ is equal to $\frac{U}{2}$ and, supposing the independence of the on-site electron energy of a spin index, we have [1] the following values for such irreducible functions:

$$
\begin{gathered}
\left.G_{2}^{(0) i r}\left(\sigma, i \omega_{1} ; \sigma, i \omega_{2} \mid \sigma, i \omega_{3} ; \sigma, i \omega_{4}\right)=\delta\left(\omega_{1}+\omega_{2}-\omega_{3}-\omega_{4}\right)\right) \\
\times \beta^{2} \mu^{2} \frac{\left[\delta\left(\omega_{1}-\omega_{4}\right)-\delta\left(\omega_{1}-\omega_{3}\right)\right]}{\left(\omega_{1}^{2}+\mu^{2}\right)\left(\omega_{2}^{2}+\mu^{2}\right)} \\
\left.G_{2}^{(0) i r}\left[\sigma, i \omega_{1} ;-\sigma, i \omega_{2} \mid-\sigma, i \omega_{3} ; \sigma, i \omega_{4}\right)\right]=\beta \mu \delta\left(\omega_{1}+\omega_{2}-\omega_{3}-\omega_{4}\right) \\
\times\left\{\frac{\beta \mu \delta\left(\omega_{1}-\omega_{4}\right)\left(1-e^{\beta \mu}\right)}{\left(e^{\beta \mu}+1\right)\left(\omega_{1}^{2}+\mu^{2}\right)\left(\omega_{2}^{2}+\mu^{2}\right)}-\frac{2 \mu \beta \delta\left(\omega_{1}-\omega_{3}\right) e^{\beta \mu}}{\left(e^{\beta \mu}+1\right)\left(\omega_{1}^{2}+\mu^{2}\right)\left(\omega_{2}^{2}+\mu^{2}\right)}\right. \\
+\frac{2 \mu \beta \delta\left(\omega_{1}+\omega_{2}\right)}{\left(\omega_{1}^{2}+\mu^{2}\right)\left(\omega_{3}^{2}+\mu^{2}\right)\left(e^{\beta \mu}+1\right)} \\
\left.-\frac{2\left[\omega_{1} \omega_{2} \omega_{3} \omega_{4}-\mu^{2}\left(\omega_{1}^{2}+\omega_{2}^{2}+\omega_{3}^{2}+\omega_{1} \omega_{2}-\omega_{2} \omega_{3}-\omega_{1} \omega_{3}\right)-3 \mu^{4}\right]}{\left(\omega_{1}^{2}+\mu^{2}\right)\left(\omega_{2}^{2}+\mu^{2}\right)\left(\omega_{3}^{2}+\mu^{2}\right)\left(\omega_{4}^{2}+\mu^{2}\right)}\right\} \\
G_{2}^{(0) i r}\left[\sigma, i \omega_{1} ;-\sigma, i \omega_{2} \mid \sigma, i \omega_{3} ;-\sigma, i \omega_{4}\right]=\beta \mu \delta\left(\omega_{1}+\omega_{2}-\omega_{3}-\omega_{4}\right) \\
\left\{\frac{\beta \mu \delta\left(\omega_{1}-\omega_{3}\right)\left(e^{\beta \mu}-1\right)}{\left[\omega_{1}^{2}+\mu^{2}\right]\left[\omega_{2}^{2}+\mu^{2}\right]\left(e^{\beta \mu}+1\right)}+\frac{2 \mu \beta e^{\beta \mu} \delta\left(\omega_{1}-\omega_{4}\right)}{\left(\omega_{1}^{2}+\mu^{2}\right)\left(\omega_{2}^{2}+\mu^{2}\right)\left(e^{\beta \mu}+1\right)}\right. \\
-\frac{2 \mu \beta \delta\left(\omega_{1}+\omega_{2}\right)}{\left(\omega_{1}^{2}+\mu^{2}\right)\left(\omega_{3}^{2}+\mu^{2}\right)\left(e^{\beta \mu}+1\right)} \\
\left.+\frac{2\left[\omega_{1} \omega_{2} \omega_{3} \omega_{4}-\mu^{2}\left(\omega_{1}^{2}+\omega_{2}^{2}+\omega_{3}^{2}+\omega_{1} \omega_{2}-\omega_{1} \omega_{3}-\omega_{2} \omega_{3}\right)-3 \mu^{4}\right]}{\left(\omega_{1}^{2}+\mu^{2}\right)\left(\omega_{2}^{2}+\mu^{2}\right)\left(\omega_{3}^{2}+\mu^{2}\right)\left(\omega_{4}^{2}+\mu^{2}\right)}\right\}
\end{gathered}
$$

The corresponding functions with a simultaneous change of all the spin indices to the opposite value are supposed to be identically equal to the initial ones because of the independence of electron energies of a spin index. 
Really, to find contribution $\mathrm{Z}^{I I}\left(x \mid x^{\prime}\right)$ of these ladder diagrams to function $Z\left(x \mid x^{\prime}\right)$ we have to extract some superfluous diagrams and, therefore, to determine a new function:

$$
\begin{gathered}
H_{2}\left[x_{1}, x_{2}^{\prime} \mid x_{2}, x_{1}^{\prime}\right]=H_{2}^{i r}\left[x_{1}, x_{2}^{\prime} \mid x_{2}, x_{1}^{\prime}\right]+G_{2}^{(0) i r}\left[x_{1}, x_{2} \mid x_{2}^{\prime}, x_{1}^{\prime}\right] \\
+\sum_{11^{\prime} 22^{\prime}} G_{2}^{(0) i r}\left[x_{1}, 1 \mid x_{2}^{\prime}, 2^{\prime}\right] G_{2}^{(0) i r}\left[2, x_{2} \mid 1^{\prime}, x_{1}^{\prime}\right] t\left(2^{\prime}-2\right) t\left(1^{\prime}-1\right) .
\end{gathered}
$$

The contribution of the second kind of the ladder diagrams to irreducible function $Z\left(x \mid x^{\prime}\right)$ is

$$
\left.Z^{I I}\left(x_{1} \mid x_{1}^{\prime}\right)=\sum_{x_{2} x_{2}^{1}} \widetilde{t}\left(x_{2}^{\prime}-x_{2}\right) H_{2}\left(x_{1}, x_{2}^{\prime} \mid x_{2}, x_{1}^{\prime}\right)\right] .
$$

The contribution of the both kinds of the ladder diagrams to the $Z\left(x \mid x^{\prime}\right)$ function is

$$
Z\left(x_{1} \mid x_{1}^{\prime}\right) \simeq Z^{I}\left(x_{1} \mid x_{1}^{\prime}\right]+Z^{I I}\left[x_{1} \mid x_{1}^{\prime}\right] .
$$

There are also more complicated diagrams that must be taken into account to obtain a more correct value of this function.

\section{The coherent potential theory}

In the given section attention is paid to the solution of the equation for oneparticle Green function in the coherent potential approximation. A special case of half-filling, when the number of electrons is equal to the lattice sites number, is considered. The following approach is taken into account: the renormalized quantity $G_{2}^{i r}$ is determined only by equation (22).

Applying expression (34) to the localized irreducible two-particle Green function, equation (22) can be solved and we obtain

$$
\widetilde{G}_{2 q}^{i r}\left[\sigma, i \omega ; \sigma, i \omega_{1} \mid \sigma, i \omega_{1} ; \sigma, i \omega\right]=\beta \frac{a\left(i \omega ; i \omega_{1}\right)\left(1-\delta\left(\omega-\omega_{1}\right)\right)}{1-a\left(i \omega, i \omega_{1}\right) \xi_{2, \boldsymbol{q}}\left[\sigma, i \omega ; \sigma, i \omega_{1}\right]},
$$

where

$$
a\left(i \omega, i \omega_{1}\right)=\frac{\mu^{2}}{\left(\omega^{2}+\mu^{2}\right)\left(\omega_{1}^{2}+\mu^{2}\right)} .
$$

On the basis of expressions (6), (21) and (40) function $\Lambda_{\sigma}(\boldsymbol{q} \mid \omega)$ in this approximation can be found as

$$
\Lambda_{\sigma}(\boldsymbol{q} \mid i \omega)=\frac{-i \omega}{\omega^{2}+\mu^{2}}-\frac{1}{N} \frac{1}{\beta} \sum_{\boldsymbol{k}_{1}} \sum_{\omega_{1}} \frac{\xi_{\sigma}\left(\boldsymbol{k}_{1}-\boldsymbol{q} \mid i \omega_{1}\right) \beta\left(1-\delta\left(\omega-\omega_{1}\right)\right)}{1-a\left(i \omega, i \omega_{1}\right) \xi_{2, \boldsymbol{k}_{1}}\left[\sigma, i \omega ; \sigma, i \omega_{1}\right]} .
$$

In order to solve equation (41), the coherent potential approximation is used. [11]. After analytical continuation $(i \omega \rightarrow E)$ we obtain the following equation:

$$
\Lambda_{\sigma}(0 \mid E)=\frac{-E}{\mu^{2}-E^{2}}+\frac{T_{\sigma}(E) a(E, E)}{1-T_{\sigma}^{2}(E) a(E, E)} .
$$


Here

$$
T_{\sigma}(E)=\frac{1}{N} \sum_{q} \frac{\xi(\boldsymbol{q})}{1-\xi(\boldsymbol{q}) \Lambda_{\sigma}(0 \mid E)} .
$$

It is convenient to determine function $g(E)$ as

$$
g(E)=\frac{1-\sqrt{1-\lambda^{2}(E)}}{\lambda(E)},
$$

where

$$
\begin{gathered}
\Lambda_{\sigma}(0 \mid E) W=2 \lambda_{\sigma}(E), \\
\lambda_{\uparrow}(E)=\lambda_{\downarrow}(E)=\lambda(E) .
\end{gathered}
$$

$W$ is the width of the band energy. Then,

$$
T_{\sigma}(E)=\frac{W}{4} g(E)\left(g^{2}(E)+1\right) ; \lambda(E)=\frac{2 g(E)}{g^{2}(E)+1}
$$

and equation (42) may be rewritten as

$$
\begin{gathered}
2 g(E)\left[1-\mu^{2} a(E, E)\left(g^{2}(E)+1\right)^{3} /\left(4 v^{2}\right)\right]= \\
-\frac{W}{2} E\left(g^{2}(E)+1\right) /\left(\mu^{2}-E^{2}\right)\left[1-\mu^{2} a(E, E)\left(g^{2}(E)+1\right)^{2} g^{2}(E) /\left(4 v^{2}\right)\right],
\end{gathered}
$$

where

$$
v=\frac{2 \mu}{W}=\frac{U}{W}
$$

In order to obtain the condition for the Mott metal-dielectric transition we put $E=0$ in (45) and then obtain

$$
2 \widetilde{g}\left(4 v^{2}-\left(\widetilde{g}^{2}+1\right)^{3}\right)=0
$$

Here

$$
\widetilde{g}=g(E=0)
$$

From equation (46) it can be seen that the critical value of Coulomb interaction is equal to $v_{c}=\frac{1}{2}$. On the condition that $v \geqslant v_{c}$ the Mott metal-dielectric transition takes place. This condition is reached on the basis of equation (22), taking into account only a contribution of the irreducible function $G_{2}^{i r}$. with parallel spins. Therefore, it is different from the one of work [2] by factor $\sqrt{3}$, in work [2] $v_{c}=\frac{\sqrt{3}}{2}$.

\section{Conclusions}

Our main concern was to investigate the properties of the systems with strong electron correlations taking into account a special ladder kind of perturbation theory's diagrams.

We have obtained integral equations that realize the generalized random phase approximation for different spin channels of the scattering processes and have determined one-particle Green function. 
One of these equations has been solved by using the coherent potential approximation.

The exact solution of the integral equations can be done only for special assumptions about the theory parameters and remains our next task.

\title{
References
}

1. M I.Vladimir, V.A Moskalenko, Teor. Matem. Fiz. 82, 428 (1990) [Theor. Math. Phys. 82, .301 (1990)].

2. S.I.Vacaru, M.I.Vladimir, V.A.Moskalenko, Theor. Matem. Fiz. 85, 248 (1990) [Theor. Math. Phys. 85, 1185 (1990)].

3. S.P. Cojocaru, V.A. Moskalenko, Teor. Matem. Fiz. 97, .270 (1993)[Theor. Math.Phys. 97, $1290(1993)]$.

4. V.A.Moskalenko, S.P.Cojocaru, M.I.Vladimir, A diagram technique for strongly interaction fermion systems. One and two-band Hubbard Models (1994), preprint ICTP, IC/94/182, Trieste, Italy.

5. V.A.Moskalenko, Teor. Matem. Fiz. 110, 308 (1997) [Theor. Math. Phys. 110 , 243 (1997)].

6. J.Hubbard, J. Proc. Roy. Soc. A 276,.233 (1963).

7. P.A.Anderson, Phys. Rev. 124,.41 (1961).

8. P.M.Slobodyan, I.V.Stasyuk, Teor. Matem. Fiz. 19, 423 (1974) (in Russian).

9. R.O.Zaitsev, Zh.Eksp.Teor.Fiz. 68, 207 (1975), ibad 70, 1100.(1976).

10. Yu.A.Izyumov, Yu.M.Skryabin, Statistical Mechanics of magnetically ordered systems. Moscow. (1987) (in Russian).

11. V.A.Moskalenko, L.Z.Kon, 3 rd General conference of the Balkan Physical Union (BPU-3) Cluj-Napoca 25 sept., 381, 6P-041 (1997).

\section{Діаграмна техніка в моделі Хаббарда. Драбинкове наближення}

\author{
В.А.Москаленко ${ }^{1,2}$, Л.3.Кон ${ }^{2}$
}

1 Об'єднаний інститут ядерних досліджень, 141980 Дубна, Росія

2 Інститут прикладної фізики, Молдова, 277028 м. Кишинів, вул. Гросул, 5

Отримано 20 березня 1998 р.

На основі узагальненої теореми Віка розроблено нову діаграмну техніку для систем з сильними електронними кореляціями. Кулонівське відштовхування електронів в моделі Хаббарда розглядається як головна частина гамільтоніана і враховується в нульовому наближенні. Матричний елемент міжвузлового перескоку електронів враховується як збурення. Досліджено одночастинкову мацубарівську функ- 
цію Гріна й отримано рівняння Дайсона. Новими елементами теорії, що характерні для даного наближення, є багаточастинкові незвідні функції Кубо. Вони обертаються в нуль, коли кулонівська взаємодія рівна нулю. Головна мета даної роботи - це сумування драбинкових діаграм, що враховують найбільш суттєві спінові і зарядові флуктуації системи. Отримані інтегральні рівняння для двох каналів розсіяння. Метод когерентного потенціалу використовується для спрощення і розв'язування цих рівнянь. На цій основі встановлена умова переходу метал-діелектрик.

Ключові слова: узагальнена теорема Віка, драбинкові діаграми, модель Хаббарда

PACS: $71.28 .+d, 71.27 .+a$ 
